\title{
Spectacle Lenses With Slightly Aspherical Lenslets for Myopia Control: Clinical Trial Design and Baseline Data
}

Junhong Chen

Affiliated Eye Hospital of Wenzhou Medical University

\section{Ran Zhuo}

Affiliated Eye Hospital of Wenzhou Medical University

Jiayan Chen

Affiliated Eye Hospital of Wenzhou Medical University

\author{
Adeline Yang \\ R\&D AMERA, Essilor International
}

\section{Ee Woon Lim}

R\&D AMERA, Essilor International

Jinhua Bao

Wenzhou Medical University

\section{Björn Drobe}

Wenzhou Medical University, Essilor International Research Center (WEIRC)

\section{Daniel P. Spiegel}

R\&D AMERA, Essilor International

Hao Chen ( $\square$ chenhao@mail.eye.ac.cn )

Wenzhou Medical University

Lijie Hou

Affiliated Eye Hospital of Wenzhou Medical University

\section{Research Article}

Keywords: myopia, spectacle lenses, prospective studies, refractive errors, axial length.

Posted Date: September 16th, 2021

DOI: https://doi.org/10.21203/rs.3.rs-847219/v1

License: (9) (1) This work is licensed under a Creative Commons Attribution 4.0 International License. Read Full License 


\section{Abstract}

Objectives: Myopia is a major public health problem and it is essential to find safe and effective means to control its progression. The study design and baseline data are presented for a one-year prospective, double-masked, cross-over, randomized clinical trial evaluating the efficacy of single vision spectacle lenses with concentric rings of slightly aspherical contiguous lenslets technology (SAL) on myopia control.

Methods: One hundred 8- to 13-year old Chinese children with a refractive error of -0.75 D to -4.75 D were assigned into two groups. In Group 1, SAL then single vision lenses were each worn for 6 months, and Group 2 wore the lenses in the reversed order. Primary outcomes are axial length and spherical equivalent of cycloplegic refractive error. Secondary outcomes include corneal thickness, anterior chamber depth, lens thickness, visual acuity, and lens adaptation.

Results: No significant differences in baseline parameters (cycloplegic spherical equivalent, axial length, age) were found between groups $(0.49<p<0.94)$. All children adapted well to the test lenses and there was no significant difference in visual acuity between the SAL and single vision lenses $(p=0.27)$.

Conclusions: The children in the two well balanced groups had comparable visual acuity and adapted well to the test lenses. These results imply the visual acuity can be well improved by SAL lens. Clear visual acuity provide the assurance for good compliance in this longitudinal study.

\section{Background}

Myopia has become a major public health problem worldwide. In the past decades, the prevalence of myopia has gradually increased. The prevalence of myopia and high myopia were $22.9 \%$ and $2.7 \%$ of the world population in 2000 , and the it is expected to rise to $49.8 \%$ and $9.8 \%$, respectively in 2050[1]. Compared with other regions, the prevalence of myopia is highest in Asia, especially in East Asia[2-4]. Lin et al.[3] reported the prevalence of myopia in Taiwan has risen from 74\% to 84\% from 1983 to 2000 in 16 to 18 years old children. Furthermore, the prevalence of high myopia in 18-year-old students increased from $10.9 \%$ to $21 \%$.

Excessive progression of myopia has been shown to be associated with sight-threatening complications[5-7]. The odds of complications increase with a higher degree of myopia and greater axial length[8]. Thus, preventing the progression of myopia and slowing down the elongation of the eye at an early stage is vital to avoid later ocular complications.

Several interventions are currently available to slow myopia progression, such as topical administration of atropine and use of orthokeratology (Ortho-K), multifocal contact lenses, or specially designed spectacle lenses. High doses of atropine are effective but associated with significant adverse effects such as photophobia and impaired accommodation. In addition, the significant rebound effect after cessation of high-concentration atropine limits its widespread use[9, 10]. Lower dosages of topical atropine (0.01\%) have less adverse effects; however, axial length control effect is limited[11]. Ortho-K is effective in myopic refractive error and myopia control[12-14], but the efficacy is significantly associated with corneal shape[15-18]. Recently, many studies have reported that dual-and multifocal soft contact lenses are effective strategies for slowing myopia progression[19-22]. However, potential ocular complications such as conjunctivitis and keratitis[23] limit the large-scale usage of soft contact lenses in myopia control.

On the other hand, spectacle lenses represent safe and easy-to-administer option for myopia control. Among spectacle lenses, (prismatic) bifocals provide the best myopia control efficacy[24]; however, aesthetics considerations hinder their more extensive usage. Progressive addition spectacle lenses (PALs) are more aesthetic, but their treatment effect is smaller[25-28]. Spectacle lens with peripheral hyperopic defocus amelioration have minimal, if any, myopia control effect[29, 30]. Recently introduced spectacle lenses with myopia control segments may be a promising avenue in myopia control combining good efficacy and aesthetics[31, 32].

Hence, a randomized double masked cross-over clinical trial was undertaken to evaluate the myopia control effect of novel spectacle lenses with contiguous slightly aspherical lenslets (SAL).

\section{Methods}

\section{Study design}


This is a prospective, double-masked, cross-over, and randomized clinical trial. The duration of the study is 13 months (see Fig. 1 for more details about the timeline of this study), and it consists of 7 visits.

Eligible subjects were randomized into two groups. Group 1 will wear SAL lenses for 6 months, followed by 6 months of wearing single vision lenses. In Group 2, the order in which the lenses are worn is reversed. In this paper, we refer to the spectacles worn during the first 6 months (regardless of lens type) as study equipment 1 and the spectacles worn during the second 6 months (regardless of lens type) as study equipment 2 (Fig. 1). At each visit, all subjects are instructed to wear the study equipment for more than seven hours every day.

The study is being conducted at the Hangzhou Branch of Zhejiang Eye Hospital Affiliated to Wenzhou Medical University. The study and its protocol follow the tenets of the Declaration of Helsinki and were approved by the institutional review board of the Eye Hospital of Wenzhou Medical University. Written informed consent was obtained from participants and their guardians after a detailed explanation about this study and the possible risks and benefits at the first visit. The study is registered at the Chinese Clinical Trial Registry (ChiCTR1900021002, 24/01/2019).

\section{Study participants}

A total of 100 children were recruited from the hospital into this study between January and March 2019. The inclusion criteria were best-corrected visual acuity equal to or better than 1.0, age between 8 and 13 years inclusive, spherical equivalent of cycloplegic autorefraction between $-0.75 \mathrm{D}$ to $-4.75 \mathrm{D}$ in each eye, astigmatism no more than $1.50 \mathrm{D}$, anisometropia no more than $1.00 \mathrm{D}$, and absence of strabismus. Subjects with a history of ocular or systemic disease, history of wearing PALs, or prior myopia control treatment were excluded from participation in the study.

The study was designed to achieve $80 \%$ power to detect a minimum difference of $0.27 \mathrm{D}$ with a SD of $0.37 \mathrm{D}$ in 6 months at a $5 \%$ level of significance. Using an online sample size calculator by Clinical \& Translational Science Institute33, the theoretical sample size was 42 with a 1:1 sample ratio in each group. Allowing a maximum drop-out rate of $15 \%$, the number to be recruited for each group was estimated to be 50 .

\section{Randomization and masking}

All subjects were assigned into the two groups (described in the Study Design section) at a 1:1 ratio using covariate-adaptive randomization[33]. A scheduled randomization was generated and children were randomly assigned by Study Manager (ESSILOR R\&D). The investigators cannot access to the randomization list.

According to the scope of responsibilities, the examiners are either masked or unmasked. The masked examiners are responsible for subjective refraction, cycloplegic autorefraction, axial length, corneal thickness, anterior chamber depth measurements and assessing visual acuity with the study equipment. The unmasked examiners are in charge of dispensing and documenting adverse events.

\section{Test lenses}

The treatment lens is a polycarbonate single vision spectacle lens with a spherical front surface with 11 concentric rings formed by contiguous slightly aspherical lenslets.

The control lens is a standard polycarbonate single vision lens.

\section{Main outcome measures}

There are two main outcome measures: the axial length $(A L)$ and the spherical equivalent (SER) of cycloplegic autorefraction. The $A L$ is measured using an optical low-coherence reflectometry device (Lenstar LS900; Haag-Streit, Koeniz, Switzerland). Five measurements are taken, and the average value per eye is used for data analysis. The refraction is measured by a closed-field autorefractors (Topcon RM-800 and Topcon 8900). The measurement is taken ten times on each eye and the mode of the sphere, cylinder, and axis per eye is recorded for data analysis. The SER is calculated as sphere $+0.5 \times$ cylinder. The SER is taken only at baseline, the fifth visit and the last visit to minimize cycloplegia during the study.

\section{Secondary outcome measures}

Corneal thickness (CT), anterior chamber depth (ACD), and lens thickness (LT) are taken with the Lenstar LS900 (Haag-Streit, Koeniz, Switzerland) together with AL. Five measurements are taken, and the average value per eye is used for further data analysis. Distance- 
corrected visual acuity (DCVA) is evaluated using a standard 100\% contrast English Early Treatment Diabetic Retinopathy Study (ETDRS) logMAR chart at $4 \mathrm{~m}$ with usual correction or new distance prescription. DCVA is scored using the standard technique of subtracting 0.02 logMAR units for each correctly identified optotype.

\section{Study equipment questionnaire}

The questionnaire contains six questions. The first four questions evaluate adaptation and subjectively evaluate various aspects of vision with the study equipment on a scale of 1 to 10 . Question 1 quantifies the clarity of vision ( $1=$ blurred, $10=$ clear); question 2 evaluates perception of ghost images $(1=$ none, $10=$ severe); question 3 evaluates satisfaction with the study equipment $(1=$ not satisfied, $10=$ satisfied), and question 4 evaluates the comfort of study equipment ( $1=$ uncomfortable, $10=$ comfortable). The remaining questions assess compliance in terms of how long the study equipment is worn (question 5: hours per day, and question 6 : days per week).

\section{Study visits and procedures}

The procedures performed at each visit are summarized in Table 1.

During the recruitment and baseline visit, we first performed a full ocular examination, including presenting visual acuity, objective and subjective refraction, anterior segment and fundus inspection, and binocular vision status (near and far phoria, near point of convergence (NPC), Worth 4-dot test), followed by acquisition of the primary outcome measures (cycloplegic SER and AL) and all secondary outcome measures (DCVA, AL, CT, ACD, and $L T$ ). The subjects chose their frame, and the same frame is used for SAL and control single vision lenses, i.e., before and after cross-over. At the end of the baseline visit, the subjects are dispensed with washout single vision lenses corresponding to the prescription for their first study equipment.

The main follow-up visits include the 6-month cross-over visit and the final 12-month visit with all primary outcome measures, including the questionnaires.

The additional study visits comprise the dispensing visit (approximately one month after the baseline), 3-month, 5-month, and 9-month visits with only non-cycloplegic AL and secondary outcome measures. In addition, at the 5-month visit, we acquire non-cycloplegic subjective refraction as a basis for the prescription for the study equipment worn during the second phase of the study (i.e., after crossover).

Between one and three days after the dispensing visit and 6-month visit, we administer the compliance and adaptation questionnaire over the phone.

During all study visits, the study equipment is adjusted, and subjects are instructed to wear it at least 7 hours per day.

Cycloplegia is always induced by $1 \%$ cyclopentolate hydrochloride (Alcon Laboratories) eyedrops delivered three times, five minutes apart after induction of corneal anaesthesia with proparacaine (0.5\% Alcaine, Alcon Laboratories).

Table 1. Visit schedule for the study. V1 to V7 represent visits 1 through 7

AL, axial length; CT, corneal thickness; ACD, anterior chamber depth; LT, lens thickness; DCVA, distance-corrected visual acuity 


\begin{tabular}{|llllllll|}
\hline Visit schedule & V1 & V2 & V3 & V4 & V5 & V6 & V7 \\
\hline Informed consent & X & & & & & & \\
\hline Eye examination & X & & & & & & \\
\hline Inclusion and exclusion criteria & X & & & & & & \\
\hline Study frame choice & X & & & & & & \\
\hline Monocular pupillary distance and fitting measurements & X & & & X & & & \\
\hline Washout spectacles delivery & X & & & & & & \\
\hline Study spectacles delivery & & X & & & X & & \\
\hline AL, CT, ACD and LT measurements & X & X & X & X & X & X & X \\
\hline Cycloplegic autorefraction & X & & & & X & & X \\
\hline Non-cycloplegic subjective refraction & X & & & X & & & \\
\hline DCVA & X & X & X & X & X & X & X \\
\hline
\end{tabular}

\section{Data analyses}

Baseline data analyses

For data showing normal distribution, paired-sample t-tests were used for within-subject interocular comparisons and independentsample t-tests were used for between-group comparisons. For data showing non-normal distribution, equivalent non-parametric tests were used. Bivariate correlations were used to evaluate the relationships between outcome measures. As the inter-eye correlation for ocular parameters is high, only the right eye data were analyzed. Statistical analyses were performed using SPSS 16.0 (SPSS Inc., Chicago, IL, USA).

Prospective data analyses

The changes in spherical equivalent cycloplegic auto-refractive error and AL between the follow-up and baseline data will be used to evaluate myopia progression. Myopia progression and axial elongation will be compared between the SAL lenses and the single vision lenses using independent-sample t-tests. A multivariate regression model will be used to evaluate the relationships between variables such as age, gender, baseline myopia, parental myopia, NPC and phoria level, and myopia progression.

\section{Results}

\section{Overall baseline data}

We recruited 102 children from the Hangzhou Branch of Zhejiang Eye Hospital. One child dropped out just after the randomization period for personal reasons. One child was excluded from the study because of his/her large magnitude of phoria that manifested before the dispensing visit. Thus, the data from 100 children (54 males and 46 females) aged 8 to 12 years old (mean age, $9.49 \pm 1.42$ years) were analysed and are reported in this paper. We found no significant difference in the SER or AL between the eyes $(p=0.27$ and $p=0.30$, respectively).

The mean AL was $24.84 \pm 0.76 \mathrm{~mm}$ (range: 23.13 to $26.86 \mathrm{~mm}$ ), and the mean SER was $-2.69 \pm 0.86 \mathrm{D}$ (range: -1.00 to $-4.50 \mathrm{D}$ ). These two measures were significantly correlated $\left(r^{2}=0.18, p<0.001 ;\right.$ Fig. 2$)$.

More details on the baseline values, including the secondary outcome measures, can be found in Table 2.

Table 2. Baseline characteristics of the subjects and interocular comparison. Data are expressed as the mean \pm SD. SER, spherical equivalent refractive error; AL, axial length; CCT, central corneal thickness; ACD, anterior chamber depth; LT, lens thickness; $t / p$, pairedsample t-tests were used to compare the values between the two eyes. 


\begin{tabular}{lllllll} 
& Age (years) & SER $(\mathrm{D})$ & $\mathrm{AL}(\mathrm{mm})$ & $\mathrm{CCT}(\mu \mathrm{m})$ & ACD $(\mathrm{mm})$ & $\mathrm{LT}(\mathrm{mm})$ \\
\hline Minimum value & 8 & -4.50 & 23.13 & 473.4 & 2.76 & 2.97 \\
\hline Maximum value & 12 & -1.00 & 26.86 & 603.6 & 3.80 & 3.61 \\
\hline Mean value & $9.49 \pm 1.42$ & $-2.69 \pm 0.86$ & $24.84 \pm 0.76$ & $545.20 \pm 26.78$ & $3.25 \pm 0.20$ & $3.32 \pm 0.15$ \\
\hline t value & - & 1.11 & -1.04 & -1.39 & 0.46 & -1.23 \\
\hline p value & - & 0.27 & 0.30 & 0.17 & 0.65 & 0.22
\end{tabular}

Between-group comparison of baseline data

Eligible subjects were randomized to two groups of 50 subjects. The mean age was $9.48 \pm 1.36$ years (range: 8 to 12 ) in Group 1 and $9.50 \pm 1.50$ years (range: 8 to 12$)$ in Group 2. There was no significant difference between the groups $(p=0.94)$.

The two groups were matched for SER and AL. The mean AL was $24.79 \pm 0.67 \mathrm{~mm}$ (range: 23.35 to $26.59 \mathrm{~mm}$ ) in Group 1 and $24.89 \pm$ $0.84 \mathrm{~mm}$ (range: 23.13 to $26.86 \mathrm{~mm}$ ) in Group 2. The mean SER was $-2.68 \pm 0.83 \mathrm{D}$ (range: -1.25 to $-4.50 \mathrm{D}$ ) in Group 1 and $-2.72 \pm 0.90$ $D$ (range: -1.00 to $-4.50 D)$ in Group 2. There was no significant difference between the groups for AL or $S E R(p=0.49$ and $p=0.83$, respectively). The SER and AL were negatively correlated in both groups (Group 1: $r^{2}=0.10, p=0.029 ;$ Group 2: $r^{2}=0.26, p<0.001 ;$ Fig. 2).

There were no significant differences between the groups for any of the secondary outcome measures $(0.29<p<0.80)$. More details on the baseline values in the two groups, including the secondary outcome measures, can be found in Table 3.

Table 3. Characteristics of the subjects by group. Data are expressed as the mean \pm SD. SER, spherical equivalent refractive error; $A L$, axial length; CCT, central corneal thickness; ACD, anterior chamber depth; $L T$, lens thickness; $t / p$, independent-sample t-tests were used to compare between-group parameters; G1, Group 1; G2, Group 2

\begin{tabular}{|c|c|c|c|c|c|c|c|c|c|c|c|c|c|c|}
\hline & \multicolumn{2}{|c|}{ Age (y) } & \multicolumn{2}{|c|}{ Gender } & \multicolumn{2}{|c|}{ SER (D) } & \multicolumn{2}{|c|}{$\mathrm{AL}(\mathrm{mm})$} & ССТ $(\mu \mathrm{m})$ & \multicolumn{2}{|c|}{$\mathrm{ACD}(\mathrm{mm})$} & \multicolumn{2}{|c|}{$\mathrm{LT}(\mathrm{mm})$} & \\
\hline & G1 & G2 & G1 & G2 & G1 & G2 & G1 C & & $\mathrm{G} 1 \quad \mathrm{G} 2$ & $\mathrm{G} 1$ & $\mathrm{G} 2$ & $\mathrm{G} 1$ & $\mathrm{G} 2$ & \\
\hline Boys & & & 27 & 27 & & & & & & & & & & \\
\hline Girls & & & 23 & 23 & & & & & & & & & & \\
\hline $\begin{array}{l}\text { Minimum } \\
\text { value }\end{array}$ & 8 & 8 & & & -4.50 & -4.50 & 23.35 & 23.13 & 473.4 & 505.4 & 2.86 & 2.76 & 2.97 & 3.05 \\
\hline $\begin{array}{l}\text { Maximum } \\
\text { value }\end{array}$ & 12 & 12 & & & -1.25 & -1.00 & 26.59 & 26.86 & 603.0 & 603.6 & 3.80 & 3.76 & 3.60 & 3.61 \\
\hline $\begin{array}{l}\text { Mean } \\
\text { value }\end{array}$ & $\begin{array}{l}9.48 \\
\pm \\
1.36\end{array}$ & $\begin{array}{l}9.50 \\
\pm \\
1.50\end{array}$ & & & $\begin{array}{l}-2.68 \\
\pm \\
0.83\end{array}$ & $\begin{array}{l}-2.72 \\
\pm \\
0.90\end{array}$ & $\begin{array}{l}24.79 \\
\pm \\
0.67\end{array}$ & $\begin{array}{l}24.89 \\
\pm \\
0.84\end{array}$ & $\begin{array}{l}545.88 \\
\pm \\
27.99\end{array}$ & $\begin{array}{l}544.53 \\
\pm \\
25.78\end{array}$ & $\begin{array}{l}3.28 \\
\pm \\
0.22\end{array}$ & $\begin{array}{l}3.23 \\
\pm \\
0.19\end{array}$ & $\begin{array}{l}3.31 \pm \\
0.16\end{array}$ & $\begin{array}{l}3.33 \pm \\
0.14\end{array}$ \\
\hline $\mathrm{t}$ value & -0.07 & & & & 0.22 & & -0.69 & & 0.25 & 1.06 & & -0 . & & \\
\hline$p$ value & 0.94 & & & & 0.83 & & 0.49 & & 0.80 & 0.29 & & 0.3 & & \\
\hline
\end{tabular}

\section{Lens adaptation}

All subjects adapted well to both SAL lenses and single vision lenses (Table 4). The mean DCVA at the dispensing visit was $0.06 \pm 0.08$ logMAR (range: -0.14 to 0.24 logMAR) in Group 1 (SAL lenses) and $0.04 \pm 0.05$ logMAR (range: -0.1 to 0.14 logMAR) in Group 2 (single vision lenses, $S V L)$, with no significant difference between the groups $(p=0.27)$.

For the perception of ghost images, we found no significant difference between the lens types (mean values: $1.04 \pm 0.20$ for SAL lenses and $1.00 \pm 0.00$ for SVL; $p=0.16$ ). Similarly, there was no significant difference for the comfort of the spectacles (mean values: $9.68 \pm$ 0.55 for SAL lenses and $9.84 \pm 0.42$ for SVL; $p=0.11$ ). Regarding clarity of vision and vision satisfaction, small but significant differences were observed between the two lens types. The subjects rated single vision lenses as providing higher clarity of vision (mean values: $9.70 \pm 0.58$ for SAL lenses and $9.93 \pm 0.25$ for $S V L ; p=0.01$ ) and vision satisfaction (mean values: $9.72 \pm 0.50$ for SAL lenses and $9.91 \pm 0.29$ for SVL: $0.19 ; p=0.02$ ). 
Table 4. Adaptation to the SAL and single vision lenses. DCVA, distance-corrected visual acuity. Data expressed as the mean \pm SD. Group 1: in phase one for Group 1, the subjects wear the SAL lenses; Group 2: in phase one for Group 2, the subjects wear single vision lenses (SVL).

\begin{tabular}{lllll} 
& SAL & SVL & t value & p value \\
\hline DCVA & $0.06 \pm 0.08$ & $0.04 \pm 0.06$ & 1.11 & 0.27 \\
\hline Perception of ghost images & $1.04 \pm 0.20$ & $1.00 \pm 0.00$ & 1.43 & 0.16 \\
\hline Comfort of spectacles & $9.68 \pm 0.55$ & $9.84 \pm 0.42$ & -1.64 & 0.11 \\
\hline Clarity of vision & $9.70 \pm 0.58$ & $9.93 \pm 0.25$ & -2.59 & 0.01 \\
\hline Vision satisfaction & $9.72 \pm 0.50$ & $9.91 \pm 0.29$ & -2.32 & 0.02
\end{tabular}

\section{Discussion}

Although numerous interventions are currently available to slow myopia progression, it still be benefit for young children to develop effective and safe strategies for myopic retardation. Spectacle lenses with contiguous aspherical lenslets may be an interesting option. At present, there are few reports on the myopia control effect of these lenses. From this perspective, we conduct a double-masked, cross-over, randomized clinical trial to assess the efficacy of spectacle lenses with rings of contiguous slightly aspherical lenslets.

We follow 100 children randomized into two groups of 50 . The two groups are well matched for the main co-variate parameters, such as age, spherical equivalent refractive error, and $\mathrm{AL}(0.49<\mathrm{p}<0.94)$, minimizing their effects on the results of the study.

In this study, we adopted a one-year cross-over design that is not very common in myopia research. We believe that this approach has merit, particularly for completely novel interventions whose myopia control effect is difficult to infer from previous studies. Unlike regular clinical trial design, this cross-over design may provide a within-subject treatment comparison with little to no influence of genetics and other subject-specific factors. However, it must be noted that these analyses should be interpreted with caution because they may be biased by possible carry-over effects or difficulty with subject masking.

We also opted to have a shorter interval (maximum of 3 months) between the study visits; that is, a maximum of 3 months versus the more standard 6 months. This decision was motivated by the interest in evaluating the myopia control effect of SAL lenses at finer temporal resolution. Although cycloplegic SER and AL are only taken at the baseline, the 6-and 12-month visits to minimize cycloplegia, we believe that these data will provide valid evidence for the effect of myopia control. More than that, the AL without cycloplegia will be taken at the 3-, 5-, and 9-month visits, these data will be informative. First, the AL is considered the important index to evaluate myopic progression [34, 35]. Second, previous research has indicated that (lack of) cycloplegia has no significant effect on AL[36]. In addition, 3-month visits may allow better compliance through parent and child engagement and, more often, equipment checks.

In general, the children adapted well to both SAL and single vision lenses. According to the results of the questionnaire, there were some small but statistically significant differences in the clarity of vision and vision satisfaction between the two spectacle lens types. Although children rated the clarity of vision and overall visual satisfaction higher with SVL, the SAL lenses did not aggravate the severity of blur or induce higher discomfort. Moreover, the mean values were above 9 in both groups and the differences were 0.23 for clarity of vision and 0.19 for vision satisfaction. Considering that the values came from a questionnaire with a 1 to 10 score, these differences are likely clinically irrelevant.

According to spontaneous verbal feedback, five of the children reported slight perception of blur in the peripheral vision, and two children reported blur when reading, but they adopted a strategy to obtain a clear image. One child felt dizzy on the first day of using the SAL lens. In all these children, the adaptation did not exceed one day, and none of them discontinued the trial because of an adaptation problem.

To ensure the accuracy of the DCVA, measurements were taken using an ETDRS logMAR chart at $4 \mathrm{~m}$ under stable room lighting conditions. Although the corrected visual acuity was slightly better with single vision lenses, we did not find a significant difference between SAL lenses and single vision lenses. The SAL lenses do not compromise DCVA.

\section{Conclusion}

Page $7 / 11$ 
In conclusion, Lenses with contiguous aspherical lenslets are an emerging opportunity for myopia control. With the exception of adaptation problem in the initial stage of using SAL lenses, this type of lens could be well adapted. If the SAL lenses are capable of slowing myopia progression, it will provide a safe and effective optical strategy option for children.

\section{Abbreviations}

SAL: Slightly aspherical contiguous lenslets technology

Ortho-K: Orthokeratology

PALs: Progressive addition spectacle lenses

AL: Axial length

SER: Spherical equivalent of autorefraction

CT: Corneal thickness

ACD: Anterior chamber depth

LT: Lens thickness

DCVA: Distance-corrected visual acuity

ETDRS: Early Treatment Diabetic Retinopathy Study

NPC: Near and far phoria, near point of convergence

SVL: Single vision lenses

\section{Declarations}

\section{Acknowledgements}

Not applicable.

\section{Author Contributions}

Hao Chen, Lijie Hou contributed to the conception of the study. Junhong Chen, Ran Zhuo and Jiayan Chen performed the study. Junhong Chen, Ran Zhuo, Adeline Yang, Ee Woon Lim contributed significantly to analysis and manuscript preparation. Junhong Chen and Ran Zhuo performed the data analyses and wrote the manuscript. Jinhua Bao, Björn Drobe, Daniel P. Spiegel helped perform the analysis with constructive discussions.

\section{Funding Sources}

This work was supported by the Project of Wenzhou Medical University under Grant KJHX1607.

\section{Data Availability Statement}

The data that support the findings of this study are not publicly available because their containing information that could compromise the privacy of research participants, but are available from the corresponding author (Lijie Hou) upon reasonable request.

\section{Ethics approval and consent to participate}

The study and its protocol follow the tenets of the Declaration of Helsinki and were approved by the institutional review board of the Eye Hospital of Wenzhou Medical University. Written informed consent was obtained from participants and their guardians after a detailed explanation about this study and the possible risks and benefits at the first visit. The study is registered at the Chinese Clinical Trial Registry (ChiCTR1900021002). 
Consent for publication

Not applicable.

\section{Conflict of Interest Statement}

Jinhua Bao is the Associate Director of Wenzhou Medical University-Essilor International Research Centre. Adeline Yang, Daniel P. Spiegel and Björn Drobe are employees of Essilor International, the company that supplied the study device and holds the following patent applications related to this work: WO2019166653 WO2019166654 WO2019166655.

No conflicts of interest exist for the rest of the authors.

Author details

1 School of Ophthalmology and Optometry and Eye Hospital, Wenzhou Medical University, Wenzhou, Zhejiang Province, China

2 Department of Optometry Center, Affiliated Eye Hospital of Wenzhou Medical University, Hangzhou, Zhejiang Province, China

3 Wenzhou Medical University - Essilor International Research Center (WEIRC), Wenzhou, Zhejiang Province, China

4 R\&D AMERA, Essilor International, Singapore, Singapore

\section{References}

1. Holden BA, Fricke TR, Wilson DA, Jong M, Naidoo KS, Sankaridurg P, Wong TY, Naduvilath TJ, Resnikoff S: Global Prevalence of Myopia and High Myopia and Temporal Trends from 2000 through 2050. Ophthalmology 2016, 123(5):1036-1042.

2. Lam CS, Goldschmidt E, Edwards MH: Prevalence of myopia in local and international schools in Hong Kong. Optom Vis Sci 2004, 81(5):317-322.

3. Lin LL, Shih YF, Hsiao CK, Chen CJ: Prevalence of myopia in Taiwanese schoolchildren: 1983 to 2000. Ann Acad Med Singapore 2004, 33(1):27-33.

4. Saw SM, Tong L, Chua WH, Chia KS, Koh D, Tan DT, Katz J: Incidence and progression of myopia in Singaporean school children. Invest Ophthalmol Vis Sci 2005, 46(1):51-57.

5. Flitcroft DI: The complex interactions of retinal, optical and environmental factors in myopia aetiology. Prog Retin Eye Res 2012, 31(6):622-660.

6. Sheu SJ, Ger LP, Ho WL: Late increased risk of retinal detachment after cataract extraction. American journal of ophthalmology 2010, 149(1):113-119.

7. Suzuki Y, Iwase A, Araie M, Yamamoto T, Abe H, Shirato S, Kuwayama Y, Mishima HK, Shimizu H, Tomita G et al: Risk factors for open-angle glaucoma in a Japanese population: the Tajimi Study. Ophthalmology 2006, 113(9):1613-1617.

8. Asakuma T, Yasuda M, Ninomiya T, Noda Y, Arakawa S, Hashimoto S, Ohno-Matsui K, Kiyohara Y, Ishibashi T: Prevalence and risk factors for myopic retinopathy in a Japanese population: the Hisayama Study. Ophthalmology 2012, 119(9):1760-1765.

9. Tong L, Huang XL, Koh AL, Zhang X, Tan DT, Chua WH: Atropine for the treatment of childhood myopia: effect on myopia progression after cessation of atropine. Ophthalmology 2009, 116(3):572-579.

10. Tan D, Tay SA, Loh KL, Chia A: Topical Atropine in the Control of Myopia. Asia Pac J Ophthalmol (Phila) 2016, 5(6):424-428.

11. Yam JC, Jiang Y, Tang SM, Law AKP, Chan JJ, Wong E, Ko ST, Young AL, Tham CC, Chen LJ et al: Low-Concentration Atropine for Myopia Progression (LAMP) Study: A Randomized, Double-Blinded, Placebo-Controlled Trial of $0.05 \%, 0.025 \%$, and $0.01 \%$ Atropine Eye Drops in Myopia Control. Ophthalmology 2019, 126(1):113-124.

12. Swarbrick HA, Alharbi A, Watt K, Lum E, Kang P: Myopia control during orthokeratology lens wear in children using a novel study design. Ophthalmology 2015, 122(3):620-630.

13. Hiraoka T, Kakita T, Okamoto F, Takahashi H, Oshika T: Long-term effect of overnight orthokeratology on axial length elongation in childhood myopia: a 5-year follow-up study. Invest Ophthalmol Vis Sci 2012, 53(7):3913-3919.

14. Cho P, Cheung SW: Retardation of myopia in Orthokeratology (ROMIO) study: a 2-year randomized clinical trial. Invest Ophthalmol Vis Sci 2012, 53(11):7077-7085. 
15. Santodomingo-Rubido J, Villa-Collar C, Gilmartin B, Gutierrez-Ortega R: Factors preventing myopia progression with orthokeratology correction. Optom Vis Sci 2013, 90(11):1225-1236.

16. Zhong Y, Chen Z, Xue F, Zhou J, Niu L, Zhou X: Corneal Power Change Is Predictive of Myopia Progression in Orthokeratology. Optom Vis Sci 2014, 91(4):404-411.

17. Zhong Y, Chen Z, Xue F, Miao H, Zhou X: Central and Peripheral Corneal Power Change in Myopic Orthokeratology and Its Relationship With 2-Year Axial Length Change. Invest Ophthalmol Vis Sci 2015, 56(8):4514-4519.

18. Lee EJ, Lim DH, Chung TY, Hyun J, Han J: Association of Axial Length Growth and Topographic Change in Orthokeratology. Eye Contact Lens 2018, 44(5):292-298.

19. Lam CS, Tang WC, Tse DY, Tang YY, To CH: Defocus Incorporated Soft Contact (DISC) lens slows myopia progression in Hong Kong Chinese schoolchildren: a 2-year randomised clinical trial. The British journal of ophthalmology 2014, 98(1):40-45.

20. Aller TA, Liu M, Wildsoet CF: Myopia Control with Bifocal Contact Lenses: A Randomized Clinical Trial. Optom Vis Sci 2016, 93(4):344-352.

21. Sankaridurg P, Holden B, Smith E, 3rd, Naduvilath T, Chen X, de la Jara PL, Martinez A, Kwan J, Ho A, Frick K et al: Decrease in rate of myopia progression with a contact lens designed to reduce relative peripheral hyperopia: one-year results. Invest Ophthalmol Vis Sci 2011, 52(13):9362-9367.

22. Paune J, Morales H, Armengol J, Quevedo L, Faria-Ribeiro M, Gonzalez-Meijome JM: Myopia Control with a Novel Peripheral Gradient Soft Lens and Orthokeratology: A 2-Year Clinical Trial. Biomed Res Int 2015, 2015:507572.

23. Solomon A: Allergic manifestations of contact lens wearing. Curr Opin Allergy Clin Immunol 2016, 16(5):492-497.

24. Huang J, Wen D, Wang Q, McAlinden C, Flitcroft I, Chen H, Saw SM, Chen H, Bao F, Zhao Y et al: Efficacy Comparison of 16 Interventions for Myopia Control in Children: A Network Meta-analysis. Ophthalmology 2016, 123(4):697-708.

25. Gwiazda J, Hyman L, Hussein M, Everett D, Norton TT, Kurtz D, Leske MC, Manny R, Marsh-Tootle W, Scheiman M: A randomized clinical trial of progressive addition lenses versus single vision lenses on the progression of myopia in children. Invest Ophthalmol Vis Sci 2003, 44(4):1492-1500.

26. Gwiazda JE, Hyman L, Norton TT, Hussein ME, Marsh-Tootle W, Manny R, Wang Y, Everett D, Grouup C: Accommodation and related risk factors associated with myopia progression and their interaction with treatment in COMET children. Invest Ophthalmol Vis Sci 2004, 45(7):2143-2151.

27. Yang Z, Lan W, Ge J, Liu W, Chen X, Chen L, Yu M: The effectiveness of progressive addition lenses on the progression of myopia in Chinese children. Ophthalmic Physiol Opt 2009, 29(1):41-48.

28. Hasebe S, Ohtsuki H, Nonaka T, Nakatsuka C, Miyata M, Hamasaki I, Kimura S: Effect of progressive addition lenses on myopia progression in Japanese children: a prospective, randomized, double-masked, crossover trial. Invest Ophthalmol Vis Sci 2008, 49(7):2781-2789.

29. Kanda H, Oshika T, Hiraoka T, Hasebe S, Ohno-Matsui K, Ishiko S, Hieda O, Torii H, Varnas SR, Fujikado T: Effect of spectacle lenses designed to reduce relative peripheral hyperopia on myopia progression in Japanese children: a 2-year multicenter randomized controlled trial. Japanese journal of ophthalmology 2018, 62(5):537-543.

30. Sankaridurg P, Donovan L, Varnas S, Ho A, Chen X, Martinez A, Fisher S, Lin Z, Smith EL, 3rd, Ge J et al: Spectacle lenses designed to reduce progression of myopia: 12-month results. Optom Vis Sci 2010, 87(9):631-641.

31. Lam CSY, Tang WC, Tse DY, Lee RPK, Chun RKM, Hasegawa K, Qi H, Hatanaka T, To CH: Defocus Incorporated Multiple Segments (DIMS) spectacle lenses slow myopia progression: a 2-year randomised clinical trial. The British journal of ophthalmology 2020, 104(3):363-368.

32. Bao J, Yang A, Huang Y, Li X, Pan Y, Ding C, Lim EW, Zheng J, Spiegel DP, Drobe B et al: One-year myopia control efficacy of spectacle lenses with aspherical lenslets. The British journal of ophthalmology 2021.

33. Frane JW: A method of biased coin randomization, its implementation, and its validation. Drug Information Journal 1998, 32(2):423-432.

34. Bullimore MA, Richdale K: Myopia Control 2020: Where are we and where are we heading? Ophthalmic Physiol Opt 2020, 40(3):254270.

35. Brennan NA, Toubouti YM, Cheng X, Bullimore MA: Efficacy in myopia control. Prog Retin Eye Res 2020:100923.

36. Huang J, McAlinden C, Su B, Pesudovs K, Feng Y, Hua Y, Yang F, Pan C, Zhou H, Wang Q: The effect of cycloplegia on the lenstar and the IOLMaster biometry. Optom Vis Sci 2012, 89(12):1691-1696. 


\section{Figures}

\section{Baseline}

Phase 1

(6 months)
Phase 2

(6 months)

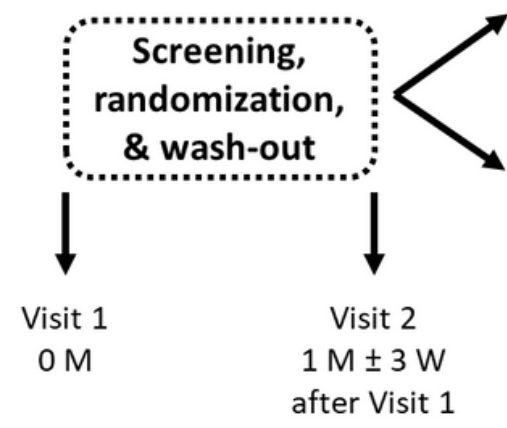

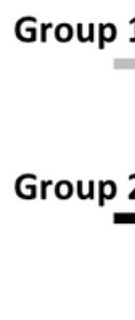

SAL

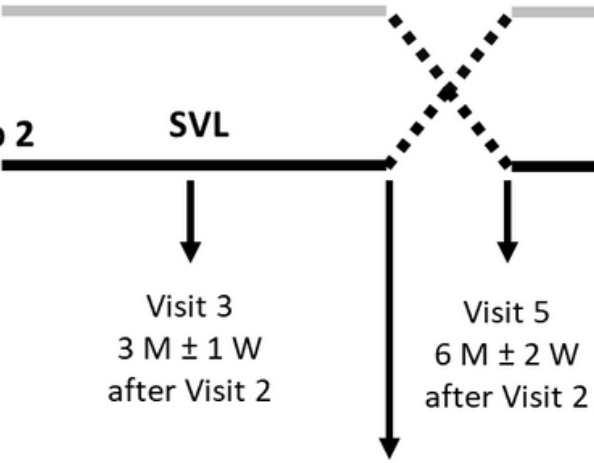

Visit 4

$5 \mathrm{M} \pm 1 \mathrm{~W}$

after Visit 2
SAL

Group 2

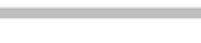

SVL
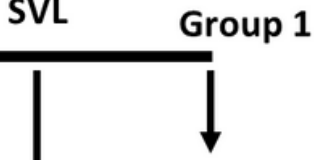

Visit 7

$12 \mathrm{M} \pm 2 \mathrm{~W}$ after Visit 2

\section{Figure 1}

Flow chart of the study; M - month(s), W - week(s)
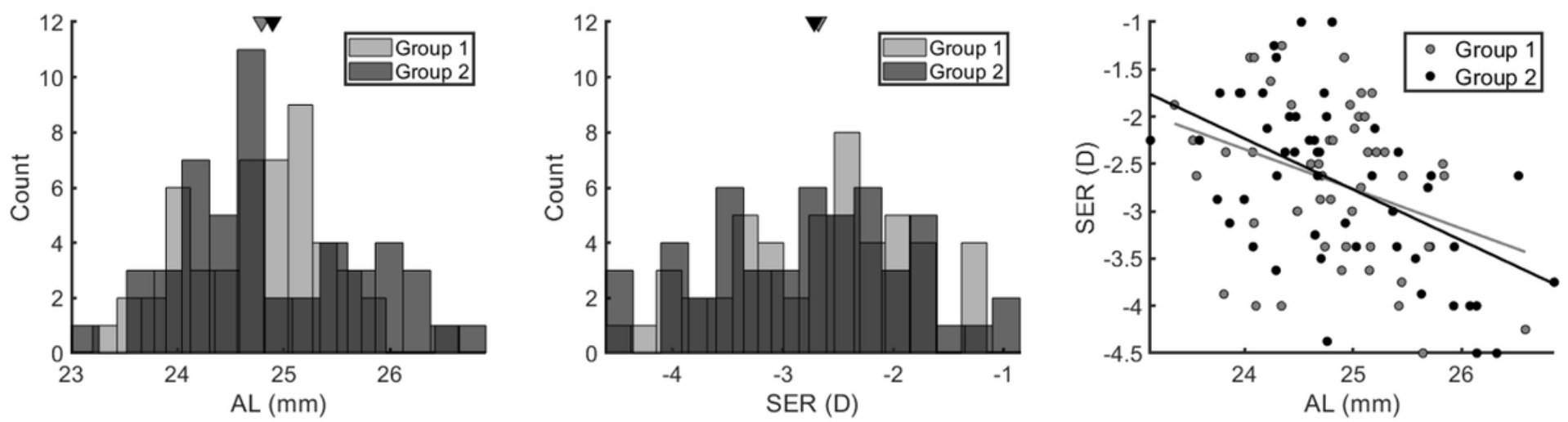

Figure 2

Distributions and relationships between the primary outcome measures at baseline. Left panel - axial length (AL), middle panel cycloplegic spherical equivalent (SER), right panel - correlations between AL and SER. Lighter shade shows Group 1 (SAL); darker shade Group 2 (single vision lens; SVL). Triangles filled with the corresponding shade indicate the groups' means. 